Chaenorhinum grandiflorum (Cosson) Willk.

Muy frecuente (WF49, WG40, WG60, WG70, WG80, WG81 y WG91, XG01, WF68). Pastizales terofíticos. Plantagini ovatae-Chaenorhinetum grandiflorii (Stipion capensis).

Linaria glauca L. Chaz subsp. aragonensis (Lange) Valdés Rara (WG80). Sobre roca de yeso.

Leontodon hispidus L. subsp. hispidus Raro (WG80). Pastizales.

Onopordon corymbosum Willk. subsp. corymbosum Poco frecuente (WG60, WF68). Ruderal viaria. Onopordetea acanthii.

Iris xiphium L.

Poco frecuente (WG80).

Juncus subulatus Forskal

Raro (WG80). Planta de lugares húmedos, más o menos salinos.

Aegilops neglecta Req. ex Bertol.

Poco frecuente (WG60). Subnitrófila. Brometalia rubenti-tectori.

Avena barbata Pott ex Link

Frecuente. (WG60, WG70, WG80, WG81, WG91, WF99). Eriales, medios viarios, lindes, cultivos. Stellarietea mediae.

Phalaris brachystachys Link Raro (WG80). Cultivos, bordes viarios y veredas.

Agradecemos a A. ASENSI MARFIL y B. DIEZ GARRETAS, del Dpto. de Botánica de la Fac. de Ciencias de Málaga, la ayuda prestada.

\title{
PLANTAS ARVENSES Y RUDERALES INTERESANTES DE LA PROVINCIA DE CORDOBA
}

\author{
A. PUJADAS \& J.E. HERNANDEZ BERMEJO \\ Departamento de Botánica \\ E.T.S.I. Agrónomos (Córdoba)
}

RESUMEN: Se relaciona un total de 39 especies de ecología arvense y ruderal. La mayor parte de ellas se citan por primera vez en la provincia de Córdoba.

SUMMARY: A number of 39 taxa recolected like weeds are considered. Most of them are new records for the province of Córdoba. 
Como resultado del estudio de la flora arvense y ruderal de la provincia de Córdoba, donde se han llegado a recolectar 941 especies de esta ecología (Pujadas, 1986) destacamos aquellas que presentan un marcado interés corológico, siendo en su mayor parte primeras citas para la provincia.

Se detalla para cada uno de los casos el lugar de recolección y su ecología con indicación de la cuadrícula U.T.M. de. $1 \mathrm{~km}$ de lado. Se incluyen sólo un máximo de tres citas para cada una de estas especies, estando respaldadas, en todos los casos por pliegos depositados en el Herbario de la Cátedra de Botánica Agrícola de la E.T.S.I.A. de Córdoba ( COA ).

Rumex tingitanus L.

Montemayor, viñedo (UG-4166), 21.IV.1981. Pujadas (COA 1194); Montoro a Cardeña, km 5, cuneta (UH-7814), 24.V.1983, Pujadas \& García-Salmones ( COA 1193).

Rumex papillaris Boiss. \& Reuter

Cabra a Carcabuey, km 10, cuneta (UG-7747), 4.VI.1981, Hernández-Bermejo \& Pujadas (COA 1166); Espiel, Sa del Castillo, plantación de almendros (UH-2127), 22.V.1984, Pujadas (COA 1165).

Rumex intermedius DC.

Entre Luque y Zuheros, talud y cunetas (UG-8558), 27.V.1984, Pujadas \& Poyato (COA 1164).

Rumex palustris Sm.

Palma del Río, en plantación de naranjos (UG-0273), 6.VII.1982, Pujadas (COA 1241); Palma del Río: Las Huertas, cuneta (TG-9974), 6.VII.1984, Hidalgo \& Saavedra.

Especie que Webb (1964:88) considera para España mientras que Jalas \& Suominen (1979:63) la omiten expresamente de nuestro territorio.

Atriplex halimus L.

Castro del Río, erial y borde de camino (UG-6722), 3.XI.1981, Pujadas ( COA 10782).

Herniaria scabrida Boiss.

Villanueva del Rey, arcén de carretera (UH-1229), 10.V.1983, Pujadas ( COA 1529).

Spergularia nicaeensis Sarato ex Burnat

Palma del Río, en cultivo de naranjo (UG-0175), 2.III.1982, Pujadas (COA 3768); Córdoba, eriales (UG-4293), 21.III.1983, Pujadas (COA 3370), Valenzuela a Baena, km 10, en cultivo de trigo (UG-9174), 23.III.1983, Pujadas (COA 3769).

Spergularia bocconei (Sheele) Graebner

Villa del Río a Cardeña, km 4, cuneta (UH-8805), 10.VI.1984, Pujadas ( COA 1635).

Silene rubella L. subsp. bergiana (Lindman) Malagarriga

Espejo, olivar (UG-5972), 16.III.1982, Pujadas (COA 1508); Sotogordo, cultivos hortícolas (UG-4537), 12.IV.1983, Pujadas (COA 1512); Encinas Reales, olivar (UG-6626), 21.IV.1983, Pujadas (COA 1513).

Ranunculus macrophyllus Desf.

Peñarroya, cuneta (UH-0140), 27.IV.1981, Pujadas (COA 1908); Iznájar, 
cuneta (UG-9315), 17.IV.1982, Pujadas (COA 1906); Doña Mencía a Nueva Carteya, km 6, olivar (UG-8447), 19.V.1982, Pujadas (COA 1907).

Papaver pinnatifidum Moris

Alcaracejos, cultivo de trigo (UH-2841), 17.V.1981, Pujadas (COA 2409); Espiel, erial (UH-2329), 15.III.1983, Pujadas (COA 2417); Villanueva del Rey, cultivo de avena (UH-1229), 10-V-1983, Pujadas (COA 2415).

Fumaria faurei (Pugsley) Lidén

Bujalance, margen de cultivo (UG-8096), 23.III.1982, Pujadas \& García-Salmones, (COA 2156); Espejo, olivar (UG-6672), 17.IV.1982, Pujadas (COA 2155); Santaella a La Carlota, km 4, cuneta (UG-3661), 12.IV.1983, Pujadas (COA 2159).

Coronopus didymus (L.) Sm.

Sotogordo, cultivos hortícolas (UG-4537), 10.III.1982, Pujadas (COA 2605); Córdoba, viaria (UG-4293), 14.IV.1982, Pujadas (COA 2600); Castro del Río, cultivos hortícolas (UG-6873), 15.VI.1983, Pujadas (COA 2603).

Especie muy frecuente pero de la que sólo conocemos para la provincia de Córdoba las referencias procedentes de inventarios publicados por Rivas Martínez (1975:137).

Vicia sativa L. subsp. cordata (Wulfen ex Hoppe) Ascherson \& Graebner Palma del Río, cultivo de naranjo (UG-0371), 12.V.1982, Pujadas (COA 3254); entre Moriles y Monturque, erial (UG-5947), 17.V.1983, (COA 3251); Montoro a Adamuz, km 7, olivar (UH-7512), 24.V.1983, Pujadas (COA 3253).

Ononis hirta Poiret

Santaella, cultivo de habas (UG-3058), 17.V.1983, Pujadas (COA 2961).

Trifolium strictum L.

Cardeña a Pozoblanco, km 3, en cultivo de cebada-avena (UH-7938), 4.V.1982, Pujadas \& Montoro (COA 3523).

Especie escasamente recolectada, en la provincia donde conocemos solamente la cita de Pau (1921:297).

\section{Euphorbia terracina L.}

Sotogordo, borde carretera (UG-4537), 15.VI.1982, Pujadas (COA 4320). Solamente conocemos las referencias de Infante (1982:456) para esta especie en la provincia.

\section{Viola kitaibeliana Schultes}

Entre Villanueva de Córdoba y Cardeña, en cultivo de cebada (UH-6939), 17.V.1981, Pujadas (COA 8157); Alcaracejos, olivar (UH-2850), 30.III.1982, Pujadas (COA 8155); Cardeña a Pozoblanco, km 12, cuneta (UH-7139), 4.V.1982, Pujadas ('COA 8153).

Solamente conocemos las citas de Pau (1921:291) para la provincia.

\section{Capnophyllum peregrinum (L.) Lag.}

El Carpio, cuneta (UH-0801), 11.V.1984, Pujadas (COA 8741); Espejo, suelos inundados temporalmente (UG-6072), 31.V.1985, Pujadas \& Poyato (COA 9550).

Ferulago brachyloba Boiss. \& Reuter

Córdoba a Villaviciosa, km 9, en olivar (UH-3903), 27.IX.1983, Pujadas (COA 3792); Hornachuelos, talud de carretera (UH-0388), 7.X.1984, Pujadas \& Poyato (COA 3788). Córdoba.

Citada anteriormente por Bernardi (1979:85) en la provincia de 
Opopanax chironium (L.) Koch

Priego de Córdoba, en talud de carretera y en olivar (UG-9944), 24.VI.1983, Pujadas (COA 3791).

Considerada como muy rara en la provincia (Muñoz, 1982:244).

Tordylium officinale L.

Posadas a Villaviciosa, km 15, cuneta (UH-1701), 24.V.1985, Pujadas ( COA 7689).

Especie que TUTIN (1968:367) no cita para la Península Ibérica.

Daucus aureus Desf.

Espejo, arvense en cultivo de girasol (UG-6172), 15.VI.1983, Pujadas (COA 3787); Santaella, cerca del Arroyo Térmico, arvense en cultivo de habas (UG-3159), Pujadas 17.V.1983, (COA 3785).

Ruiz de Clavijo \& Cabezudo (1979:44) indican por primera vez su presencia en Andalucía Occidental (Sevilla) y Muñoz (1982:226) la cita en Córdoba.

Centaurium spicatum (L.) Fritsch Espejo, arvense en girasol (UG-5873), 5.VII.1984, Pujadas (COA 9086).

\section{Lavandula multifida L.}

Montoro, cuneta y talud (UH-7909), 24.V.1983, Pujadas \& García Salmones (COA 3878).

Citadá en la provincia por Fernández-Corrales (1984:333) considerándola como muy rara.

Solanum villosum Miller, subsp puniceum (Kirschleger) Edmons Palma del Río, en cultivos hortícolas (TG-9874), 2.X.1984, Pujadas ( COA 7021).

Verbascum simplex Hoffmanns \& Link

Belalcázar, cuneta (UH-0973), 27.V.1983, Pujadas (COA 7478); entre Conquista y Torrecampo, cuneta (UH-8356), 26.IV.1983, Pujadas (COA 7479); Córdoba a Villaviciosa de Córdoba, km 20, margen de carretera (UH-3309), 9.VI.1983, Pujadas (COA 7477).

\section{Verbascum pulverulentum Vill.}

Villa del Río a Cardeña, km 7, borde de cultivo (UH-8910), 24.V.1983, Pujadas \& García Salmones (COA 3959).

Especie rara en Andalucía Occidental, recolectada anteriormente en Córdoba por Fernández Corrales (1984:339).

Kickxia spuria (L.) Dumort. subsp. integrifolia (Brot.) R. Fernandes Priego de Córdoba a Baena, km. 7, plantación de membrilleros (UG-9649), 28. IX.1982, Pujadas (COA 7491).

Orobanche haenseleri Reuter Cardeña a Villa del Río, $\mathrm{km}$ 5, en cuneta parasitando Vicia lutea (UH-8633), 10.VI.1984, Pujadas \& Poyato (COA 7340).

Orobanche amethystea Thiull.

Posadas a Palma del Río, km 9, cuneta parasitando Daucus carota subsp. maximus (UG-0785), 12.V.1982, Pujadas (COA 7343); Palma del Río, Las Huertas, plantación de naranjo, parasitando Eryngium campestre (UG-0371), 12.V.1982, Pujadas (COA 7348);Villanueva del Rey, margen cultivo, parasitando Carthamus lanatus, (UH-1229), 10.V.1983, Pujadas (COA 7347).

Carduncellus cuatrecasasii G. López

Bélmez, margen de carretera (UG-0739), 26.V.1982, Hernández-Bermejo \& 
Montoro (COA 4988); La Granjuela, margen de cultivo (TH-9545), 10.V.1983, Pujadas (COA 4986); Espiel, Sa del Castillo, en plantación de almendro (UH-1928), 22.V.1984, Pujadas (COA 5147).

Especie que había sido citada solamente en el sur de la provincia por Rivas Goday \& Rivas Martínez (1968:195).

Asphodelus aestivus Brot.

Cardeña a Villa del Río, $\mathrm{km} \mathrm{15,} \mathrm{talud} \mathrm{de} \mathrm{carretera} \mathrm{(UH-8922),}$ 10.VI.1984, Pujadas (COA 11925).

Especie de la que sólo se conocen para la provincia las citas de Pau $(1921: 296)$.

Colchicum lusitanum Brot.

Entre Espejo y Montilla, cuneta y talud de carretera (UG-6066), 6.X.1984, Pujadas \& Poyato (COA 6444).

Allium paniculatum L.

Palma del Río, Las Huertas, arvense en plantación de naranjos (UG-7064); 12.V.1981, Pujadas (COA 3898); Nueva Carteya a Castro del. Río, km 6, arvense en olivar (UG-7064), 19.V.1982, Pujadas (COA 3900); Adamuz a Montoro, km 10, arvense en olivar (UH-7313), 22.VI.1982, Pujadas (COA 3901).

Pastor \& Valdés (1983:85) no citan esta especie para la provincia de Córdoba.

Avena barbata Pott subsp. atherantha (C. Presl) Rocha Alfonso

Villaviciosa, olivar (UH-2419), 6.IV.1982, Pujadas (COA 6199); Los Biázquez, olivar (TH-8654), 15.III.1983, Pujadas (COA 6201); entre Conquista y Torrecampo, cuneta (UH-6356), 26.IV.1983, Pujadas (COA 6204).

Aira cupaniana Guss. 6625).

Venta de Azuel, olivar (UH-8343), 10.VI.1984, Pujadas \& Poyato (COA

Stipa gigantea Link

Cardeña a Pozoblanco, km 3, arvense en cebada-avena (UH-7938), 4.V.1982, Pujadas (COA 6605); Córdoba a Villaviciosa, km 16 , margen carretera (UH-3605), 9.VII.1983, Pujadas (COA 6604); Cardeña a Villa del Río, km 5, cuneta (UH-8633), 10.VI.1984, Pujadas (COA 6602).

Carex depressa Link

La Guijarrosa, margen de carretera (UG-3565), 2.III.1985, Pujadas y Poyato (COA 6879).

\section{BIBLIOGRAF IA}

BERNARDI, L. -1979- Tentamen revisionis generis Ferulago. Boissiera $30: 1-182$.

FERNANDEZ CORRALES, P. -1984- Catálogo florístico de la cuenca hidrográfica del río Bembézar. Tesis Doctoral. Córdoba.

INFANTE, F. -1982- Flora de interés farmacológico de la cuenca baja del Genil. Tesina de Licenciatura. Córdoba.

JALAS, J. \& SUOMINEN, J. -1979-Atlas Florae Europeae, 4. Helsinki.

MUÑOZ, J. -1982- Catálogo florístico de las Sierras Subbéticas de la provincia de Córdoba. Tesis Doctoral. Córdoba.

PASTOR, J. \& VALDES, B. -1983- Revisión del género Allium (Liliaceae) en la Península Ibérica e Islas Baleares. Publicaciones de la Universidad de 
Sevilla. Sevilla.

PAU, C. -1921- Diez días en Sierra Morena. Mem. Real Acad. Soc. Esp. Hist. Nat. , 287-298.

PUJADAS, A. -1986- Flora arvense y ruderal de la provincia de Córdoba. Tesis Doctoral. Córdoba.

RIVAS GODAY, S. \& RIVAS MARTINEZ, S. -1968- Matorrales y tomillares de la Península Ibérica. Suplemento n 2. Acerca de los Carthamo-Carduncellus de la Ononido-Rosmarinetea peninsular. Anales Inst. Bot. Cavanilles 25:188-197.

RIVAS MARTINEZ, S. -1975- Sobre la nueva clase Polygono-Poetea annuae. Phytocoenología. 2:123-140.

RUIZ DE CLAVIJO, E. \& CABEZUDO, B. -1979- Aportaciones a la flora de la provincia de Sevilla. Lagascalia 9:39-49.

TUTIN, T.G. -1968- Tordylium, in T.G. TUTIN \& al. (eds.) Flora Europaea 2:367. Cambridge.

WEBB, D.A. -1964- Rumex, in T.G. TUTIN \& al. (eds.) Flora Europaea 1:82-89. Cambridge.

\title{
NOTA SOBRE LA FLORA DE LA SIERRA DE CABO DE GATA (ALMERIA)
}

\author{
F. SANZ FABREGA \& M. COSTA TENORIO \\ Departamento de Botánica y Fisiologia Vegeta1 \\ Facultad de Biología \\ Universidad Complutense de Madrid
}

RESUMEN: Se citan 26 táxones para el piso termomediterráneo de la provincia de Almería. De ellos 5 se mencionan por primera vez para ésta provincia. Otros muchos suponen una ampliación de su área conocida en esta provincia. Se comenta también su situación ecológica.

ABSIRACT: Twenty six thermic mediterranean taxa recorded for Almería. Five of them are mentioned for the first time en this province. Many others suppose an enlargement of their known area. We comment also their ecology.

En esta nota recogemos una serie de táxones de éste territorio seleccionados por su interés, tanto ecológico como corológico. Todo el territorio corresponde al huso $30 \mathrm{~S}$. Los testimonios se encuentran depositados en el Herbario de la Facultad de Biología de la Universidad Complutense (MAC).

El área objeto de estudio se encuentra en la región mediterránea, superprovincia mediterráneo-iberolevantina. Pertenece al séctor almeriense de la provincia murciano-almeriense. Toda ella se encuentra incluida en el piso bioclimático termomediterráneo; el ombroclima oscila entre el semiárido y el árido. Los sustratos son de tipo volcánico con numerosos enclaves de materiales sedimentarios terciarios que ocupan las depresiones internas de las pequeñas sierras. 